\title{
Doppler resilience evaluation of different encoding techniques for underwater acoustic ranging systems
}

\author{
Santiago Murano, Carmen Pérez-Rubio \\ Electronic Department \\ University of Alcalá \\ Alcalá de Henares, Spain \\ santiago.murano@edu.uah.es, \\ mcarmen.perezr@uah.es
}

\author{
Joaquín Aparicio \\ Department of Informatics \\ University of Oslo \\ Oslo, Norway \\ joaqapar@ifi.uio.no
}

\author{
Carlos De Marziani \\ Electronic Department \\ National University of \\ Patagonia San Juan Bosco \\ Comodoro Rivadavia, Argentina \\ marziani@unpata.edu.ar
}

\begin{abstract}
Underwater acoustic communication or localization systems can be affected by the Doppler effect when the receiver or emitter is in motion. In those cases, the use of an a appropiate encoding/modulating scheme play a pivotal role in the time of arrival estimations, avoiding the need of complex auxiliary Doppler compensation algorithms. This paper presents a Doppler resilience evaluation of Zadoff-Chu based schemes, modulated by phase modulation (QPSK), Orthogonal Frequency Division Multiplexing (OFDM) and Frequency Hopping Spread Spectrum (FHSS) modulation schemes. They are also compared to traditional binary Kasami codes modulated by BPSK. An underwater acoustic propagation model has been used to assessed the aforementioned schemes, to which the Doppler effect modeling has been also incorporated.
\end{abstract}

Index Terms-Underwater ranging, Doppler effect, ZadoffChu sequences, sequence design, Ray-Tracing propagation model.

\section{INTRODUCTION}

The increase in the exploitation of marine resources, whether through extractive activities centered on fishing, hydrocarbon resources and possible off-shore exploitation, has led to an increase in the risk of pollution and deterioration of the environment. To minimize the negative impact produced by these activities, it is of great importance the monitoring of the environmental and biological variables that may be affected by them. Sensor networks deployment and the location of autonomous monitoring robots require of underwater positioning systems. Since the use of electromagnetic waves is not possible due to their fast attenuation, acoustic communication or positioning systems [1] are a better option. Based on the emission of encoded acoustic signals, most of these systems relay on the estimation of time of arrivals (TOA) for distance measurements. Furthermore, the underwater acoustic channel is one of the most complicated wireless channels due to their notable characteristics, such as long propagation delays, multi-path propagation and Doppler shift effects. In particular, the Doppler effects caused by the relative motions between emitter and receiver have a significant impact on the transmitted signals. The choice of codes and modulation schemes robust enough to overcome the extreme conditions of underwater environments has become an essential task on communication and positioning systems. The use of Zadoff-
Chu (ZC) sequences [2] has been of great interest in these environments due to their excellent correlation properties [3].

The following work presents a comparison of four encoding/modulation schemes using ZC codes modulated by Quadrature Phase Shift Keying (QPSK), Orthogonal Frequency Division Multiplexing (OFDM), and two Frequency Hopping Spread Spectrum (FHSS) schemes: Fast-FH and Slow-FH. The results are compared with traditional binary Kasami codes modulated in BPSK. Besides, the Doppler effect was incorporated to a previous underwater propagation model based on ray-tracing [4], [5]. The main objective is to assess the performance of thes schemes under the Doppler effect on shallow waters, using parameters from the Argentinian coasts of San Jorge Gulf, which constitutes a good example of marine exploitation in this kind of environments. The results show the potential benefits of using the ZC-QPSK or ZC-OFDM schemes to perform mobile robot localization based on a TOA range system.

\section{ENCODING AND MODULATION SCHEMES}

The use of encoded signals in underwater ranging systems allows to achieve high accuracy in determining traveling times, and improves the immunity to noise. Modulation is required to fulfill the spectral requirements of the transducers. This section describes the encoded/modulating schemes under analysis.

\section{A. Zadoff-Chu codes}

Zadoff-Chu (ZC) sequences, are polyphasic and unitary complex valued codes. According to [2], a $\mathrm{ZC}$ sequence $Z_{r_{k}}$ of length $L_{Z C}$, is defined as

$$
Z_{r_{k}}[l]= \begin{cases}\exp \left(j \pi \frac{r_{k} l^{2}}{L_{Z C}}\right), & \text { if } L_{Z C} \text { is even } \\ \exp \left(j \pi \frac{r_{k} l(l+1)}{L_{Z C}}\right), & \text { if } L_{Z C} \text { is odd }\end{cases}
$$

where $r_{k}=1,2, \ldots, L_{Z C}-1$ is an integer and co-prime to $L_{Z C}$ that represents the generation root for each of the $K_{Z C}=L_{Z C}-1$ available sequences.

The periodic Auto-Correlation (AC) function of a $\mathrm{ZC}$ sequence has zero sidelobes as indicated in [2]. On the other hand, the aperiodic correlation of $\mathrm{ZC}$ sequences is not ideal, 


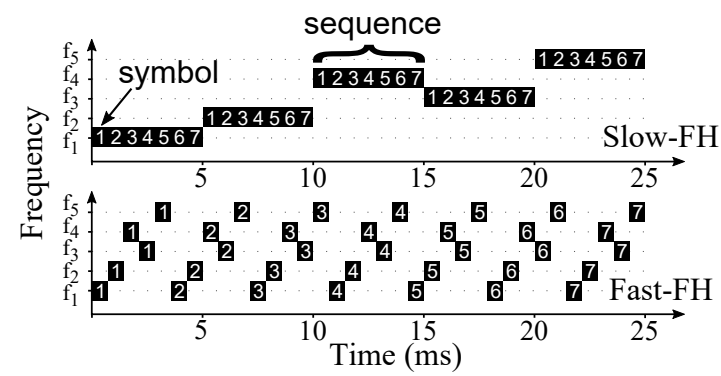

Fig. 1: FHSS hopping pattern example describing Fast-FH and Slow-FH for a single ZC sequence of length 7 , and five hops (carriers $f_{1}$ to $f_{5}$ ).

but achieves low correlation sidelobes [6], which is desired on range estimation applications.

To fulfill the spectral requirements of the ultrasonic transducer, four modulation schemes are studied in an underwater environment for ZC codes:

1) QPSK modulation: $\mathrm{ZC}$ sequences are complex valued. Thus, one way to transmit these codes is based on a Quadrature Phase Shift Keying (QPSK) scheme, where the real and imaginary parts are phase modulated by using orthogonal carriers, such as cosine and sine wave signals. Then, the emitted signal will be the sum of both.

2) OFDM modulation: Orthogonal Frequency Division Multiplexing (OFDM) modulation is a multicarrier medium access technique that distributes data over equally spaced frequency carriers in the bandwidth of the transducer. This modulation scheme can be efficiently implemented by using the Inverse Fast Fourier Transform (IFFT) at the emitter, and the FFT at the receiver. Because of the properties of $\mathrm{ZC}$ sequences, its FFT results in another ZC sequence with similar properties. To allocate the Zadoff-Chu code in the appropriate band defined by the transducer response, each code bit has to be fed into the channels of the IFFT that correspond to the desired output frequencies, and the rest of the channel inputs should be filled with zeros [7].

3) Frequency Hopping Spread Spectrum Modulation: In FHSS modulation, the signal to be transmitted is propagated using a carrier that alternates or "hops" from one carrier to another following a pattern, so that the signal remains in the band for short periods of time. The carriers are obtained like in the QPSK modulation. In the current study, two forms of frequency hopping were considered: Slow-FH and FastFH [7]. In Slow-FH, the entire ZC sequence is transmitted before changing the carrier. In contrast, Fast-FH hops for every element of the sequence. Fig. 1 shows an example with five silmultaneous emitters and a ZC code with 7 elements.

These modulation schemes have already been evaluated on airborne indoor ultrasonic positioning systems [7], whereas Kasami sequences were also evaluated for underwater communication systems, but they were not compared to Zadoff-Chu [8].

\section{B. Kasami sequences BPSK modulated}

Kasami sequences [9] are widely employed in location or range systems [10], [11]. They belong to the family of Pseudo Noise (PN) sequences and are composed by $K_{k a s}=2^{N_{k a s} / 2}$ binary sequences, with length $L_{k a s}=2^{N_{k a s}}-1$, where $N_{k a s}$ is an even natural number. An easy way to transmit these binary codes is by means of a BPSK modulation with one or more carrier cycles to modulate each bit in the code $K A S_{k}[n]$, so the final phase 0 or $\pi$ will depend on the bit value.

\section{UNDERWATER PROPAGATION MODEL}

An acoustic propagation model has been used to simulate the emission from one emitter to one receiver in underwater environments and to test the performance of the aforementioned encoding schemes. Several mathematical techniques can be used, such as ray-tracing, normal-mode models or the parabolic equation [12]. For this work the ray-tracing model fully described in [5] has been used. This model takes in consideration the common phenomenology on this kind of environments, such us: sound speed profile dependence on temperature, transmission loss due to propagation in the medium, as well as the bounce on the sea surface or the bottom, according to a bathymetric profile. Finally, it considers additive white Gaussian noise added to the received signal.

\section{A. Doppler effect description}

Here, we have extended the model in [5] with the Doppler effect produced by the relative movement between the receiver and the transmitter. This motion effect changes the frequency of the sound waves propagating through the channel, causing the transmitted signal's length to shrink or expand.

To add this effect to the propagation model, a virtual sampling frequency $f_{s}^{\prime}$ was considered for the emitted signal [11]:

$$
f_{s}^{\prime}=f_{s}[1-\frac{\overrightarrow{v_{r}}}{c} \overbrace{\frac{\overrightarrow{r_{e}}-\overrightarrow{p_{r}}}{\left|\vec{r} \bar{e}-\overrightarrow{r_{r}}\right|}}^{=1}]=f_{s}\left[1-\frac{\overrightarrow{v_{r}}}{c}\right]
$$

where $f_{s}$ is the actual sampling frequency, $c$ is the speed of sound in water, $\overrightarrow{r_{r}}$ is the receiver position vector, $\overrightarrow{r_{e}}$ is the emitter position vector, and $\overrightarrow{v_{r}}$ is the receiver velocity vector. From this frequency, the signal acquired by the receiver at the actual sampling frequency is obtained by an interpolation and decimation process. Both the emitter and the receiver are placed at the same depth, which results in a constant sound speed $c$. On the other hand, the emitter is located in a fixed position whereas the receiver is moving away from it.

\section{B. Configured parameters}

The configured parameters have been chosen considering real measurements on the coast of San Jorge Gulf in Patagonia, Argentina, which is a weak multipath environment. Simulation results can be compared with experimental tests in a future research. Table I summarizes the configuration parameters. 
TABLE I: Configuration parameters for the propagation model.

\begin{tabular}{lc}
\hline & Shallow water parameters \\
\hline Water density & $1024 \mathrm{~kg} / \mathrm{m}^{3}$ \\
Bottom density & $1941 \mathrm{~kg} / \mathrm{m}^{3}$ \\
Bottom s. speed & $1749 \mathrm{~m} / \mathrm{s}$ \\
Wind speed & $8.33 \mathrm{~m} / \mathrm{s}(30 \mathrm{~km} / \mathrm{h})$ \\
Temperature & $15^{\circ} \mathrm{C}$ \\
$\mathrm{pH}$ & 7 \\
Salinity & $33.24 \mathrm{ppt}$ \\
SNR & $15 \mathrm{~dB}$ \\
Emitter depth & $15 \mathrm{~m}$ \\
Receiver depth & $15 \mathrm{~m}$ \\
Separation & $200 \mathrm{~m}$ \\
\hline
\end{tabular}

The wind speed, was set at $30 \mathrm{~km} / \mathrm{h}$ considering it as the annual average wind speed. This value also reduce the multipath produced by the bounce in the surface, a way to isolate the Doppler effect.

To compare the different encoded-modulation schemes under evaluation, the time duration of the emitted signal was fixed at $20.5 \mathrm{~ms}$ approximately, setting as emitter a Neptune T257 ultrasonic transducer [13] with a central frequency of 25 $\mathrm{kHz}$ and $18 \mathrm{kHz}$ bandwidth. A model of this transducer has been considered to filter the emitted signal according to its frequency response. The sampling frequency for the emission is $f_{s}=500 \mathrm{kHz}$. The encoding and modulation schemes are generated as follows:

1) Zadoff-Chu - QPSK (ZC-QPSK): A ZC sequence with length $L_{Z C}=257$, QPSK modulated with an oversampling factor $O_{f}=20$ and two cycles per symbol. Emission time of $t_{e}=20.56 \mathrm{~ms}$.

2) Zadoff-Chu - OFDM (ZC-OFDM): A ZC sequence with length $L_{Z C}=283$, OFDM modulated between 18 and $32 \mathrm{kHz}$. Emission time $t_{e}=20.22 \mathrm{~ms}$.

3) Zadoff-Chu - Fast Frequency Hopping (ZC-FastFH): A ZC sequence with length $L_{Z C}=7$, a QPSK symbol of length 290 samples, five total hops, emission time $t_{e}=20.3 \mathrm{~ms} .1$ hop per symbol (see Fig. 1).

4) Zadoff-Chu - Slow Frequency Hopping (ZC-SlowFH): A ZC sequence with length $L_{Z C}=7$, a QPSK symbol of length 290 samples, five total hops, emission time $t_{e}=20.3$ ms. 1 hop per 7 symbols (see Fig. 1).

5) Kasami - BPSK (Kas-BPSK): A Kasami sequence of length $L_{k a s}=255$, BPSK modulated with an oversampling factor $O_{f}=20$ and two cycles per symbol. Emission time of $t_{e}=20.4 \mathrm{~ms}$.

\section{Simulation RESUltS}

This section describes the obtained results for the evaluated schemes under the Doppler effect, where a relative movement between the emitter and the receiver at constant speed was considered. Figs. 2 (a) to (e) depict the ambiguity function for the proposed schemes on the modeled shallow waters of San Jorge Gulf, revealing their performance against Doppler effect. The constant speed is increased between 0 and $4 \mathrm{~m} / \mathrm{s}$ at steps of $0.1 \mathrm{~m} / \mathrm{s}$.

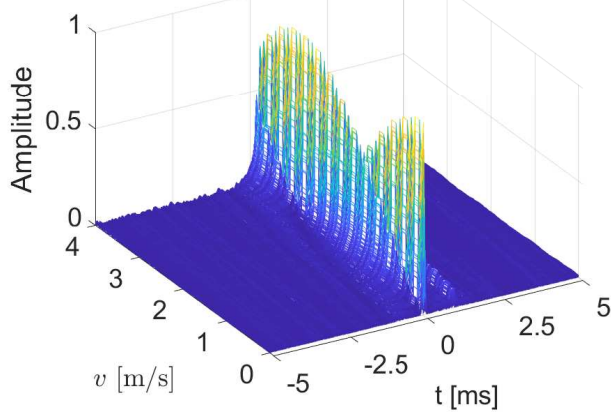

(a) Emitted signal: ZC-QPSK

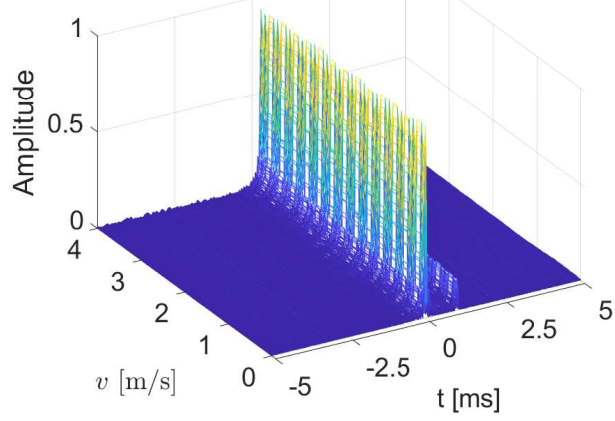

(b) Emitted signal: ZC-OFDM

Fig. 2: Ambiguity function of the received signal, with the receiver in motion.

The results show that the $\mathrm{ZC}$ based schemes exhibit a higher robustness to Doppler shift, except for ZC-FastFH scheme (Fig. 2 (d)). The Kasami encoded scheme exhibits the worst performance in terms of Doppler resilience due to the decrease of the correlation peak with the speed increment (Fig. 2 (e)).

Furthermore, Fig. 3 depicts the absolute error in the correlation peak estimation. It can be observed that it increases linearly with the relative speed since the correlation peak suffers a time deviation due to the signal length expansion. Also, it is possible to see the considerable error increment for ZC-FastFH and Kas-BPSK schemes, because of the side lobe increment and correlation peak attenuation respectively. It may be mentioned that for a better appreciation of the figure, the yaxis was zoomed-in between 0 and $1 \mathrm{~m}$. The high values that appear for ZC-FastFH and Kas-BPSK corresponds to cases in which a high secondary peak is detected instead of the mainlobe (see Figs. 2 (d) and (e)). Something similar occurs in the ZC-SlowFH scheme, at speeds higher than $3.5 \mathrm{~m} / \mathrm{s}$ a sidelobe is detected instead of the main peak. Nevertheless, the sidelobe position is close to the expected ideal main peak position what results in a reduction of the error (see Fig. 2(c)).

\section{CONCLUSIONS AND FUTURE WORKS}

A ray-tracing based underwater propagation model have been used to evaluate the Doppler resilience of Zadoff-Chu complex sequences modulated by phase (ZC-QPSK), orthogonal frequency (ZC-OFDM) and frequency hopping spread 


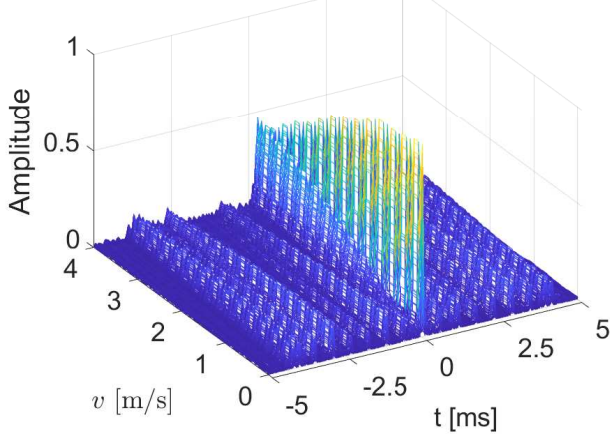

(c) Emitted signal: ZC-SlowFH

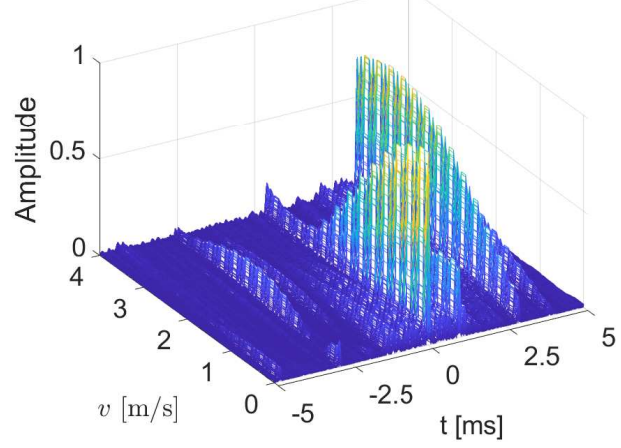

(d) Emitted signal: ZC-FastFH

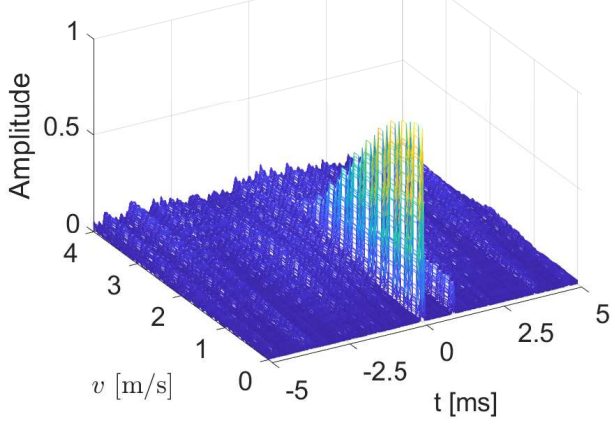

(e) Emitted signal: Kas-BPSK

Fig. 2: (continued).

spectrum (ZC-FastFH and ZC-SlowFH) modulation schemes. Moreover, they were compared with traditional Kasami sequences BPSK modulated. The simulated results considering shallow water parameters with low multipath effect, have shown better Doppler robustness when ZC-QPSK and ZCOFDM schemes were used. Kasami sequences show a significant attenuation of the correlation peak, even though their results are good enough for relative speeds lower than 1.5 $\mathrm{m} / \mathrm{s}$. Further works will consider multiple access environments with multiple emitters, a more sophisticated Doppler model considering the speed profile or Doppler compensation algorithms with a priori information about the moving device and experimental tests.

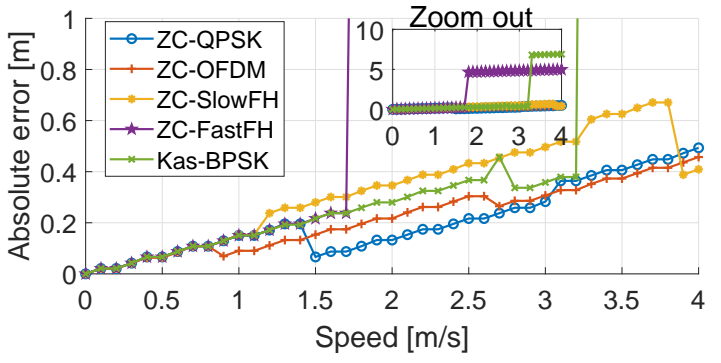

Fig. 3: Absolute error of the correlation detection with Doppler effect as a function of the receiver speed.

\section{ACKNOWLEDGMENT}

This work has been possible thanks to the support of the Spanish Ministry of Economy and Competitiveness (MICROCEBUS project, ref. RTI2018-095168-B-C51) and National University of Patagonia San Juan Bosco (Ref. SCyT 80020170200057UP).

\section{REFERENCES}

[1] A. Burguera, G. Oliver, and Y. González, "A measurement model for mobile robot localization using underwater acoustic images," in 2010 IEEE 15th Conference on Emerging Technologies \& Factory Automation (ETFA 2010). IEEE, 2010, pp. 1-4.

[2] D. Chu, "Polyphase codes with good periodic correlation properties (Corresp.)," IEEE Transactions on Information Theory, vol. 18, no. 4, pp. 531-532, 1972.

[3] X. Yu, Y. Wang, and X. Guan, "Doppler Scale Estimation for Underwater Acoustic Communications Using Dual Zadoff-Chu Sequences," in 2018 IEEE International Conference on Signal Processing, Communications and Computing (ICSPCC). IEEE, 2018, pp. 1-5.

[4] J. Aparicio, F. J. Álvarez, J. Ureña, A. Jiménez, C. Diego, and E. García, "Swell effect in shallow underwater acoustic communications," in 2010 IEEE 15th Conference on Emerging Technologies \& Factory Automation (ETFA 2010). IEEE, 2010, pp. 1-4.

[5] J. Aparicio, A. Jiménez, F. J. Álvarez, J. Ureña, C. De Marziani, D. de Diego, N. Cruz, and H. Campos, "Accurate detection of spreadspectrum modulated signals in reverberant underwater environments," Applied Acoustics, vol. 88, pp. 57-65, 2015.

[6] W. Mow and S.-Y. Li, "Aperiodic autocorrelation properties of perfect polyphase sequences," in Singapore ICCS/ISITA'92.'Communications on the Move'. IEEE, 1992, pp. 1232-1234.

[7] S. Murano, M. C. Pérez, J. Ureña, C. J. Bleakley, and C. De Marziani, "Comparison of Zadoff-Chu Encoded Modulation Schemes in an U1trasonic Local Positioning System," in 2018 International Conference on Indoor Positioning and Indoor Navigation (IPIN), Sep. 2018, pp. 206-212.

[8] J. Aparicio, "Evaluation of spread-spectrum signals in realistic underwater noisy environments," in OCEANS 2016-Shanghai. IEEE, 2016, pp. 1-9.

[9] T. Kasami, "Weight distribution formula for some class of cyclic codes," Coordinated Science Lab. University of Illinois, Tech. Rep. AD0632574, Abril 1966.

[10] J. Ureña, A. Hernández, J. J. García, J. M. Villadangos, M. C. Pérez, D. Gualda, F. J. Álvarez, and T. Aguilera, "Acoustic local positioning with encoded emission beacons," Proceedings of the IEEE, vol. 106, no. 6, pp. 1042-1062, 2018.

[11] J. A. Paredes, T. Aguilera, F. J. Álvarez, J. Lozano, and J. Morera, "Analysis of Doppler effect on the pulse compression of different codes emitted by an ultrasonic LPS," Sensors, vol. 11, no. 11, pp. 1076510784, 2011

[12] H. Ji, X. Xu, X. Guo, S. Ye, J. Chen, and X. Yang, "Direct FVM Simulation for Sound Propagation in an Ideal Wedge," Shock and Vibration, vol. 2016, 2016.

[13] Neptune Sonar Ltd. Communication Transducer T257. [Accessed: June2019]. [Online]. Available: http://www.neptune-sonar.co.uk/?s=t257 